\title{
Hallmarks for early recognition of anti-N-methyl-D-aspartate receptor (anti-NMDAR) encephalitis: A case report and review of literature
}

\author{
Victor Molina-Lopez ${ }^{* 1}$, Diego Gonzalez-Bravo ${ }^{2}$, Erika Watts-Oquendo ${ }^{2}$, Jorge Cruz-Cruz ${ }^{2}$ \\ ${ }^{1}$ Department of Internal Medicine, Veteran Affairs Hospital, San Juan, Puerto Rico \\ ${ }^{2}$ Department of Internal Medicine, University of Puerto Rico School of Medicine, Medical Sciences Campus, San Juan, Puerto \\ Rico
}

Received: November 8, 2017

DOI: $10.5430 /$ crim.v5n1p9
Accepted: December 14, 2017 Online Published: December 20, 2017

URL: https://doi.org/10.5430/crim.v5n1p9

\begin{abstract}
Anti-N-methyl-D-aspartate receptor (Anti-NMDAR) encephalitis is an under-recognized progressive neurological disorder caused by antibodies against the GluN1 (NR1) subunit of NMDA receptors in the brain. It is characterized by the subacute development of neuropsychiatric symptoms, movement disorders and seizures, often evolving into a severe and disabling encephalopathy with detrimental consequences over the functionality of affected individuals. The variable clinical presentation and infrequency with which it is clinically encountered presents a formidable diagnostic challenge in primary care. Initial misdiagnosis as psychiatric disease or infectious encephalitis significantly delays diagnosis and treatment of this medical condition. We present the case of a 34-year-old male who was evaluated after being transferred from a psychiatric institution due to worsening neuropsychiatric symptoms and seizures. Symptoms were preceded by new onset headaches, followed by behavioral changes, movement disorders, and a myriad of symptoms that pointed towards a psychiatric disorder. The non-specific nature of the presenting signs and symptoms of this patient led to initial misdiagnosis and delay of treatment. Thus, recognizing key elements of the patient's clinical presentation and progression are of paramount importance. Our aim is to highlight key clinical and diagnostic findings that could help clinicians consider anti-NMDAR encephalitis in the differential diagnosis of patients presenting similarly as our patient did, as well as to present a review of literature of this unique neurological condition.
\end{abstract}

Key Words: Anti-N-methyl-D-aspartate receptor encephalitis, Autoimmune encephalitis, Paraneoplastic encephalitis

\section{INTRODUCTION}

Anti-N-methyl-D-aspartate receptor (Anti-NMDAR) encephalitis is an under-recognized neuropsychiatric disorder caused by antibodies against the GluN1 (NR1) subunit of NMDA receptors. Despite being one of the most common and most studied autoimmune encephalitis syndromes, its variable clinical presentation continues to entail a formidable diagnostic challenge. In recent years, the number of iden- tified cases of anti-NMDAR encephalitis has risen significantly, perhaps due to increased awareness. Notwithstanding, the clinical presentation of anti-NMDA-receptor encephalitis has remained broadly under-recognized by medical professionals in primary care, especially in the early stages of the disease. In this case report, we hope to provide a comprehensive discussion of key elements that may aid clinicians to undergo early detection and management of patients with

\footnotetext{
*Correspondence: Victor Molina-Lopez; Email: victor.molina2@upr.edu; Address: Internal Medicine Residency Program, VA Caribbean Healthcare System, Office 274, 10 Casia Street, San Juan, Puerto Rico.
} 
similar presentation to our patient.

\section{CASe PResentation}

A 34-year-old healthy Hispanic male was admitted through the emergency department after being transferred from a psychiatric hospital for evaluation of worsening neurological status, behavioral changes and seizures. His neurological symptoms were preceded by new onset headaches 4 weeks prior to evaluation. Headaches were of dull-aching holocephalic nature without radiation, progressively worsening, and ranging from 7 to 9 out of 10 in intensity, and with lowto-moderate relief after NSAID analgesics. There was no significant aggravation of headache with Valsalva maneuvers, position changes, physical activity, alcohol consumption or sexual activity. He reported increasing severity of nausea without vomiting, fever or diarrhea. He denied photophobia, phonophobia, throbbing pain, or lacrimation. There were no close sick contacts, pets or history of recent travel. During a week's time, the episodes became more frequent and disabling, which prompted a visit to a local primary care community clinic. About 8 days after the onset of headaches, family members noticed behavioral changes consistent with "irrational anxiety", which prompted a second visit to the community clinics. A head CT scan without intravenous contrast was performed and showed no intracranial acute pathologies that could explain the patient's symptoms. Headache was attributed to sinusitis due to suggestive findings on CT and he was discharged home on oral amoxillin/clavulanate and supportive care. By day 10, his wife noticed confusion and paranoia. By day 11, he presented behavior consistent with visual and auditory hallucinations. His wife also witnessed brief, repetitive lateral horizontal deviations of the right eye with right lower face twitching. Subsequently, he started developing waxing-and-waning episodes of "abnormal body movements", occasional "absent" stares, and sporadic un-triggered "rage fits". By day 14, he was brought to an emergency department due to a convulsive episode at home. There were no known triggers, sweating, pallor, lightheadedness, blurred vision, or aura. During the spell, there was ictal crying, forced eye closure, head moved side to side, seizure lasted a few minutes, and movements were non synchronous. There was no tongue biting, cyanosis, mouth frothing or loss of sphincters. Awareness seemed to be preserved during the episode. The episode was initially ruled as an acute psychotic illness and the patient was transferred to a psychiatric facility, in which he spent 5 days with worsening symptoms despite optimal psychiatric treatment. During this period, he developed generalized muscle weakness with increased deep-tendon reflexes, diffuse fasciculations involving mostly the trunk as well as the proximal limb muscles, and worsening of ocular and oral dyskinesia with tongue tremors that were undulating in quality.

The patient was transferred to our institution due to persistent neurocortical symptoms with muscular weakness. He was afebrile with vital signs within normal limits. Mental status exam showed preserved orientation, but with dysphasia, perseverance, slow mentation, and paradoxical response to stimuli (e.g. intermittent mutism and catatonia, absent eye contact or visual tracking with preserved oculocephalic reflexes). There was persistent tongue tremor, orolingual dyskinesia, and intermittent right lower facial fasciculation. Deep tendon reflexes were brisk throughout with muscle fasciculation on proximal appendicular muscles and muscle strength grade of 4 . There was no Babinski, Hoffman, clonus, or meningeal signs present. Testicular exam was within normal limits for age. The rest of the physical exam upon admission was within normal limits.

\subsection{Investigations}

Complete blood panel and renal function panel were unremarkable. CPK was markedly elevated at a level of 5,600 U/L. Initial head CT scan without intravenous contrast performed at admission ( 21 days after symptom onset) showed no specific findings suggestive of viral or limbic encephalitis. Cerebrospinal fluid (CSF) analysis showed clear and colorless fluid, with opening pressure of $15 \mathrm{mmHg}$, white blood cell $23 / \mu 1$ with $100 \%$ lymphocytic pleocytosis, red blood cell $0 / \mu 1$, protein $23 \mathrm{mg} / \mathrm{dl}$, glucose $53 \mathrm{mg} / \mathrm{dl}$. Gram stain and viral meningitis panel tests of CSF were negative. EEG performed at day 22 showed continuous delta wave slowing with $1.5-$ to $2-\mathrm{Hz}$ rhythmic spike-wave activity predominating over the right hemisphere. At the time EEG was performed, the patient had active psychosis and orofacial dyskinesia. Head MRI performed at day 22 showed no specific white matter changes or structural abnormalities suggestive of viral or limbic encephalitis on TW2, FLAIR or DWI. Chest and abdominopelvic CT showed no radiologic evidence of underlying malignancy. Scrotal sonogram was unremarkable. Anti-cardiolipin Ab, anti-double-stranded DNA Ab, anti-nuclear Ab, anti-Ro/SSA and anti-La/SSB Ab, rheumatoid factors, Anti-Hu and Anti-Yo Ab, Anti-ribosomal $\mathrm{P} A b$, anti-DNase-B Ab, anti-cardiolipin IgG, IgA and IgM, anti-TPO Ab, and anti-streptolysin were found nonreactive in serum.

\subsection{Treatments and outcome}

The patient was started on a 7-day course of intravenous acyclovir $10 \mathrm{mg} / \mathrm{kg}$ every 8 hours for empiric coverage of the most common cause of viral encephalitis (Herpes Simplex Virus). Levetiracetam $1 \mathrm{~g}$ IV every 12 hours was provided at 
the beginning presumably for what by description was concerning for convulsive episodes. In addition, 5-day course of $1 \mathrm{gm}$ of intravenous methylprednisolone daily was initiated as empiric treatment for autoimmune encephalitis. Acyclovir was discontinued after completing 5 days, by which time CSF HSV PCR results came back negative. After $24 \mathrm{hrs}$ of methylprednisolone therapy, progressive interval improvement of tongue tremors, orolingual dyskinesia, verbal fluency as well as ability for self-feeding were noted. Behavioral symptoms improved significantly as well. DTRs, peripheral muscle fasciculations, and muscle strength also improved to normal after 48 hours. After completing methylprednisolone therapy, a 5 -day course of $0.4 \mathrm{~g} / \mathrm{kg}$ of IVIg for 5 consecutive days was started. Neuromuscular symptoms improved markedly with therapy. Patient became able to sit on the bed and stand up with help. Slow psychomotor activity and catatonia persisted after completing first line immunotherapy but symptoms such as disorganized behavior and thought process, as well as cognitive impairment improved remarkably. Serum and CSF samples resulted positive for anti-NMDAR IgG antibodies against GluN1 (NR1) 7 days after initiating empiric steroid treatment.

Given his remarkable positive response to high dose steroids and IVIG, a maintenance therapy with $1 \mathrm{mg} / \mathrm{kg}$ per day of oral prednisone for 3 months was initiated, to be followed by a slow conservative steroid tapering regimen with oral prednisone for a period of 8 months. Initially, we planned to initiate the chronic oral immunosuppressant therapy with oral Azathioprine $250 \mathrm{mg}$ daily as glucocorticoid sparing agent to sustain disease remission. Nonetheless, at his 2-month follow-up visit, his thiopurine methyltransferase (TPMT) activity resulted decreased, which can increase the risk of developing severe, life-threatening myelotoxicity. Thus, low dose oral mycophenolate mofetil $250 \mathrm{mg}$ twice daily was initiated. Since he tolerated dose well at his 4-month follow-up visit, dose was increased to oral $500 \mathrm{mg}$ twice daily. Given his diagnosis of autoimmune encephalitis, he will be continued on immunosuppression therapy for 1 year and malignancy screening every 3-5 years.

At his 1-month follow-up visit, he demonstrated a mRS of 4. In addition, he had a Mini Mental Status Exam (MMSE) of 28/30, showing problems with calculations. Also, he had a Montreal Cognitive Assessment (MOCA) of 28/30, showing problems with delayed recall. Catatonic and psychotic features had resolved. At his 2-month follow-up visit, he had a mRS of 3 with no improvement on MMSE or MOCA exams. He was undergoing physical therapy and was able to walk around the house with a walker for short distances and required a wheelchair for long distances. There were no new seizure events but reported persistent insomnia. At

Published by Sciedu Press his 4-month follow-up visit, he had a mRS of 1 with MMSE and MOCA of 30/30. He had also returned to his previous employment and was able to carry out all usual activities despite mild weakness. Up to 4 months after initiation of immunotherapy, he did not have any recurrences.

\section{Discussion}

\subsection{Clinical presentation}

Anti-NMDAR encephalitis has been most commonly identified in females (63\%-91\%) and young patients (37\%-40\% for $<18$ years). Male incidence has been reported to be greater in patients younger than 12 years $(37 \%)$ and older than 45 years $(43 \%)$. The median age for the disorder has been estimated to be around 23 years. ${ }^{[1-4]}$

Most patients develop a characteristic stage-like progression of symptoms. Within the first 4 weeks of the disease, most develop a similar spectrum of symptoms and severity regardless of age: $87 \%$ develop multiple symptoms while only $1 \%$ are expected to remain mono-symptomatic, $87 \%$ reach high severity in the modified Rankin Scale (mRS), and 77\% may require ICU care. ${ }^{[2]}$

For most patients, the initial symptoms consist of a nonspecific infectious-like prodrome, followed by psychiatric and neurological disturbances that may be severe enough to require ICU admission. It is this variable spectrum of initial symptoms that entails the most significant hurdle for early diagnosis, especially when a psychiatric disorder or an infectious etiology of encephalitis is entertained.

Recent estimates suggest that $57 \%$ of patients are initially misdiagnosed with psychosis, viral encephalitis or other diseases. ${ }^{[3]}$ In like manner, it is estimated that $77 \%$ of patients are initially evaluated by psychiatrists due to behavioral changes, while $23 \%$ are initially evaluated by neurologists due to neuropsychiatric symptoms. In effect, it typically takes 3 weeks before these patients are admitted to their hospital and another 2 more weeks before the correct diagnosis is made. ${ }^{[1]}$

Prodromal symptoms are usually variable, with some patients presenting a nonspecific viral-like illness, headaches or low-grade fever. The presence of febrile symptoms are nonspecific and have been variably reported in anti-NMDAR encephalitis. $^{[1,3]}$ Thomas et al. described that among 253 patients with acute febrile encephalopathy, only 2 were positive for anti-NMDA-receptor antibodies. ${ }^{[5]}$ When compared with viral etiologies of encephalitis such as Varicella-Zoster Virus (VZV), Herpes Simplex Virus type 1 (HSV-1), West Nile virus (WNV), and enteroviral encephalitis, Gable et al. demonstrated that fever was nearly omnipresent in VZV and WNV cases, whereas it was seen in only $56 \%$ of anti- 
NMDAR encephalitis patients. ${ }^{[6]}$

Headaches have also been variably reported in anti-NMDAR encephalitis. A study by Schanking et al. suggests that there may be an association with new-onset headaches and positive CSF titers of anti-NMDAR antibodies. Among the patients with new onset headaches, few met criteria for tension-type headache and migraine but most did not. Headaches were usually either continuous or, if episodic, manifested with frequent attacks of short duration ( $<4$ hours), with intensity being severe ( $>6$ of 10 on the verbal rating scale), of pressing quality, interfering with daily routine, and without migraine-typical features. Headache responded well to pain medication as well as to standard treatment of autoimmune encephalitis (steroids, immunoadsorption, plasmapheresis, rituximab). ${ }^{[7]}$

Prodromal symptoms are commonly followed by more severe neurological symptoms such as seizures, behavioral changes and movement disorders after 2-3 weeks. Females and adult patients have a tendency to present with initial psychiatric complaints, in comparison males and younger patients $(<$ 12 year of age), who tend to develop seizures initially. ${ }^{[2,3]}$ Adolescents and young adults tend to present with a combination of both. Pediatric patients have a tendency for early development of dyskinesia and movement disorders, but such findings are common in adults as well. Seizures are present in about $76 \%$ of patients with positive anti-NMDA-receptor antibodies. ${ }^{[2]}$ Notably, new-onset seizures are often the initial symptoms in children, and pose a problem when differentiating them from non-epileptic abnormal movements (choreoathetosis, oro-facio-lingual dyskinesias, pseudo-rhythmic arm and leg movements, oculogyric crisis, or opisthotonos). ${ }^{[8]}$ When compared with viral etiologies, seizures are more common in anti-NMDAR cases $(69 \%$ vs $20 \%$ of VZV encephalitis cases, which has the highest risk among the viral encephalopathies). ${ }^{[6]}$ The seizures can be variable and are typically generalized tonic-clonic seizures $(30 \%)$, partial complex (10\%), secondary generalized seizures, refractory status epilepticus, focal motor seizures, epilepsia partialis continua, or unclassified seizures. ${ }^{[1]}$

Non-epileptic convulsions or psychogenic non-epileptic seizures (PNES) can also occur. ${ }^{[9]}$ Our patient showed a clinical presentation consistent with PNES as per descriptions of family members who witnessed the event in two occasions. For such reason, Levetiracetam was discontinued after first-line immunotherapy was provided and patient showed positive clinical response. Despite discontinuation of antiepileptic drugs (AED), no further seizure-like episodes occurred. In addition, it is important to stress the point that the gold standard for diagnosis of PNES is continuous video
EEG, which was not available at our institution during his admission period to confirm this diagnosis. The onset of PNES are often gradual in character, at times may be triggered by stress, auditory or visual stimuli, and may have organic and psychosomatic etiologies. Unfortunately, there is no single biomarker that can successfully differentiate between PNES and epileptic seizures. ${ }^{[10]}$

Psychiatric symptoms and behavioral abnormalities often overshadow other neuropsychiatric symptoms. ${ }^{[2,6,7]}$ The most common initial behavioral changes are anxiety, agitation, delusional or paranoid thoughts, and visual or auditory hallucinations. These symptoms usually evolve to loss of consciousness, progression to a catatonic-like state with periods of akinesia alternating with agitation, and paradoxical responses to external stimuli. Some may have absent or inconsistent eye contact or visual tracking. The psychiatric findings are consistent NMDAR antagonism. Low dose antagonism of these receptors has been associated with psychosis, anxiety, memory disturbance, and speech dysfunction. In contrast, high dose antagonism can lead to dissociative anesthesia, catatonic features, orofacial and limb dyskinesias, autonomic instability, and seizures. This is correlated to current evidence suggesting a strong connection between schizophrenic symptoms associated with executive function and reasoning deficits, the dorsolateral prefrontal cortex of the brain and glutamate dysfunction. ${ }^{[11]}$

A recent study by Steiner et al. investigated the prevalence of serum anti-NMDAR antibodies from 459 patients with acute schizophrenia, major depression (MD) and borderline personality disorder (BLPD). Anti-NMDAR antibodies were identified in 15 of the seropositive patients, primarily in those with an initial schizophrenia diagnosis (9.9\%) as opposed to MD (2.8\%). Two patients who were initially classified as having catatonic or disorganized schizophrenia were reclassified as having anti-NMDAR encephalitis. ${ }^{[12]}$ Another study Tsutsui et al. showed that Anti-NMDA-receptor antibody has been detected in patients with various psychotic and sleep symptoms such as narcolepsy with psychotic features in patients that lack any noticeable clinical signs of encephalitis throughout the course of the disease. ${ }^{[13]}$ Mild or incomplete forms of NMDA-encephalitis may present with predominant or isolated psychiatric symptoms, leading to patients being treated in psychiatric institutions with poor success due to fulfillment of psychiatric DSM V criteria and lack of encephalitis signs. ${ }^{[6,14]}$

Furthermore, perhaps the most important differential diagnosis to consider for the evaluation and management of antiNMDAR encephalitis is the possibility of viral encephalopathy, which may share presenting features and can occur con- 
comitantly. In addition, it is necessary to consider that antiNMDAR encephalitis has been reported to be significantly more frequent than HSV-1, WNV and enteroviral encephalitis. ${ }^{[6]}$ In a study by Pruss et al, anti-NMDA-receptor antibodies were found in serum or CSF of $44(30 \%)$ HSV encephalitis patients. ${ }^{[15]}$

It is important to note that our patient developed muscular weakness, brisk tendon reflexes, and diffuse fasciculations involving the trunk and proximal limb muscles. These acute neuropathic findings showed a rapid response to immunosuppressive therapy, which points towards an autoimmune etiology. Some studies have been able to identify anti-NMDAR encephalitis cases that overlap with acute polyneuropathies and demyelinating diseases such as neuromyelitis optica spectrum disorder (NMOSD), brain stem encephalitis, leukoencephalopathy following herpes simplex encephalitis, and acquired demyelination syndromes. Also, in patients with NMOSD and overlapping anti-NMDA receptor encephalitis, coexisting antibodies antibodies against aquaporin-4 (AQP4) and myelin oligodendrocyte glycoprotein (MOG) have been identified. Other coexisting antibodies include anti-Alpha-amino-3-hydroxy-5-methyl-4isoxazolepropionic acid (AMPA) receptor antibody and anti$\mathrm{Hu}$ antibody. In addition, since NMDA receptors are also present in the anterior horn cells of the spinal cord, antibody damage on motor neurons can lead to motor neuron injury. Thus, the pathogenic circulating antibodies are able to affect not only the CNS but also the peripheral nervous system. Upper motor neuron degeneration leads to muscle spasticity, while lower motor neuron degeneration leads to muscle weakness, atrophy and twitching. Therefore, it is important to arrange diagnostic tests like nerve conduction studies and electromyography in patients with suspicion of a peripheral nervous system involvement and autoimmune encephalitis. This in turn can guide further autoimmune antibody detection studies for overlapping syndromes. ${ }^{[16]}$

\subsection{Diagnostic studies}

\subsubsection{CSF analysis}

Perhaps the most important initial screening study for patients with neurocortical symptoms suggestive of CNS pathology is a lumbar puncture with CSF analysis. CSF findings of anti-NMDAR encephalitis usually show mild to moderate lymphocytic pleocytosis (96\%), oligoclonal bands (65\%), or both. The hallmark CSF consists of lymphocytic pleocytosis of 23 white blood cells $/ \mathrm{mm}^{3}$ during the illness, which is significantly lower median value than cases of viral etiologies. ${ }^{[17,18]}$ Usual values found in other important viral etiologies are 70 white blood cells/mm3 in enterovirus and 78 white blood cells $/ \mathrm{m}^{3}$ in HSV-1. White blood cell levels in
CSF are substantially higher for WNV $\left(189\right.$ cells $\left./ \mathrm{mm}^{3}\right)$ and VZV cases $\left(167\right.$ cells $/ \mathrm{mm}^{3}$ ). CSF protein levels are also significantly lower in anti-NMDAR encephalitis cases (median of $24 \mathrm{mg} / \mathrm{dl}$ ), compared with enterovirus ( $34 \mathrm{mg} / \mathrm{dl}$ ), HSV $(52 \mathrm{mg} / \mathrm{dl})$, and VZV $(69.5 \mathrm{mg} / \mathrm{dl})$ encephalitis. Glucose values tend to be within normal range and do not differ from that of viral encephalitis. ${ }^{[6]}$

\subsubsection{Imaging studies}

Brain magnetic resonance imaging (MRI) abnormalities are nonspecific for patients with autoimmune encephalitis. Patients with seizures should be evaluated with brain MRI. It is superior to head CT and may show transient changes that include DWI changes or increased FLAIR in hippocampus, neocortex, corpus callosum, and posterior thalamus.

Autoimmune etiologies predominantly have non-specific white matter changes, which are more likely to be detected on TW2 or FLAIR than on DWI. Compared to infectious causes of encephalitis, the presence of limbic changes is more characteristic for an immune etiology than an infectious. ${ }^{[19,20]}$ MRI findings are substantially higher in HSV-1 (100\%), VZV (100\%) and WNV (60\%) encephalitis than in cases of NMDAR encephalitis $(46 \%){ }^{[6,21]}$

Brain imaging with PET or SPECT has shown diverse areas of regional of altered metabolism in patients with NMDAR and other autoimmune encephalitis syndromes but whether it can be used to identify a particular form of encephalitis or rule in or rule out autoimmune causes remains controversial. $^{[21,22]}$

\subsubsection{Electroencephalography}

The electroencephalogram (EEG) is a valuable resource for the diagnosis and evaluation of multiple neurological conditions. In general terms, EEG is most important in patients with impaired consciousness or altered mental state. It may help demonstrate evident functional disturbances in presence of normal structural imaging or it could help detect focal or lateralized abnormalities that could suggest a structural basis for an encephalopathy.

Patients with anti-NMDAR encephalitis benefit from EEG performed early in the psychotic phase. EEGs are abnormal in the acute phase of the disease and most commonly present with prominent background slowing early in the psychotic phase. It is considered the earliest and most reliable abnormal EEG finding since it is present in almost all patients. The EEG patterns in this disease represent slow neuronal network oscillations that result from downregulation of NMDA receptor-mediated synaptic function due to antibody-induced receptor internalization and reduction in NMDA surface receptor density. ${ }^{[23]}$ 
As the disease progresses, the slow wave changes become non-specific, and cannot be used to differentiate from other forms of encephalitis. Although not specific, NCSE, FIRDA, delta brush pattern, and continuous theta-delta slowing with delta paroxysms in EEG can direct diagnosis towards a possible autoimmune etiology. An EEG finding of abnormal generalized or predominantly frontotemporal slow activity in the delta-theta range is the most prevalent abnormality in anti-NMDAR encephalitis patients, as seen in $77 \%$ of patients. It is also the most frequent EEG pattern seen in HSV-1 $(50 \%)$ and WNV (75\%) encephalitis as well. ${ }^{[6]}$ Epileptic activity is present in about $23 \%$.

The most specific EEG pattern seen in anti-NMDAR encephalitis patients is the Extreme Delta Brush (EDB), which is observed in about one third of patients but has $100 \%$ specificity. This distinctive EEG pattern should prompt testing for NMDAR antibodies. The EDB pattern is characterized by diffuse generalized slowing delta activity at $1-3 \mathrm{~Hz}$ with superimposed bursts of rhythmic $12-$ to $30-\mathrm{Hz}$ activity 'on top' of the slow delta waves. ${ }^{[24-27]}$ While highly specific for NMDAR encephalitis, it may be present in other types of encephalitis as well. ${ }^{[28]}$ In addition, its presence should raise concern for higher mortality rate, more prolonged hospitalization, increased days of continuous EEG monitoring, and worst prognosis. ${ }^{[25]}$ Our patient showed continuous delta wave slowing with 1.5 - to $2-\mathrm{Hz}$ rhythmic spike-wave activity predominating over the right hemisphere. While not as common as bilateral delta wave slowing, unilateral or focal wave slowing can be observed as well.

\subsubsection{Anti-NMDAR antibodies}

Anti-NMDAR antibodies are neuronal surface-directed antibodies against postsynaptic neurons throughout the CNS Only the IgG autoantibody response against NR1 subunit of NMDA receptors has been found to be pathogenic. Both the $\operatorname{IgM}$ and $\operatorname{IgA}$ responses have no established role in diagnosing autoimmune encephalitis. ${ }^{[29]}$ About $10 \%$ of the population shows similar NMDAR-NR1-Ab seroprevalence, immunoglobulin class distribution ( $\operatorname{IgM}, \operatorname{IgA}$ and $\operatorname{IgG}$ ) and titer range in both health and disease. ${ }^{[30,31]}$

When considering the diagnostic value anti-NMDAR1 IgG autoantibody, titers obtained from CSF samples have $100 \%$ sensitivity and specificity, compared to about $85 \%$ sensitivity in serum. ${ }^{[2]}$ Thus, when CSF titer results are negative, antiNMDAR encephalitis can be ruled out. Positive ANA and nonspecific cytoplasmic staining may obscure the detection of anti-NMDAR antibodies and may produce indeterminate results in serum samples.

The use of titers for NMDAR autoantibodies to guide treatment has limited clinical utility but can be complementary.
Higher Ab titers have been found to be associated with worst outcome or the presence of teratomas. Also, titer changes in CSF seem to be more closely related to relapses than in serum but its use is impractical for assessment of clinical worsening. ${ }^{[32]}$ In addition, the use of titer to assess recovery is controversial since CSF titers continue to elevated despite clinical resolution of symptoms.

\subsection{Tumor screening}

While there does not seem to be any presenting clinical difference between idiopathic and tumor-positive paraneoplastic anti-NMDAR encephalitis, the neurological and psychiatric symptoms commonly precede the diagnosis of tumors. Tumors may be too small to be detected with conventional imaging when the neurological symptoms begin, so screening is typically done on initial presentation and repeated at increasing intervals. It is estimated that the younger the patient, the less likely a tumor will be detected and only $5 \%$ of male patients older than 18 will have an identified tumor. ${ }^{[2,22]}$ Titulaer et al. demonstrated that a tumor was identified in $38 \%$ of anti-NMDA receptor encephalitis patients, of which $97 \%$ were females between 12 to 45 years, with only $6 \%$ being $<12$ years. Only $3 \%$ of tumors were identified in males. Of the tumors identified in females, $94 \%$ were ovarian teratomas (Asians 45\%, African Americans 48\%, Caucasians $31 \%$, and Hispanics 27\%). Other tumors identified were extraovarian teratomas $(2 \%)$, and the remaining $4 \%$ were from lung cancer, breast cancer, testicular cancer, ovarian carcinoma, thymic carcinoma, or pancreatic cancer. Tumors other than teratomas are rare, but are preferentially found in males and patients older than 45 years. ${ }^{[2]}$ Lung cancer and testicular cancers are the most commonly associated with NMDA encephalitis in males. To the best of our knowledge, few case reports have documented anti-NMDAR encephalitis in male patients with underlying malignancy and most suggest idiopathic etiologies. Nevertheless, tumor removal is an essential therapeutic step and lack of early identification of an underlying malignancy can be detrimental for long-term prognosis.

Tumor-screening guidelines for paraneoplastic syndromes suggest performing sequential tumor-specific imaging studies for the most common identified malignancies in recent literature. If no tumor is found with available methods, close oncological follow-up every 3-6 months for at least 5 years is suggested. ${ }^{[33]}$ Clinical relapse should trigger repeated tumor screening. To the best of our knowledge no clear guidelines exist for syndromes with synaptic or neuronal cell-surface antibodies, thus they are currently screened similarly to syndromes with other onconeural antibodies. 


\section{Treatment and prognosis}

Like many forms of encephalitis, early treatment is of paramount importance. Whether or not a tumor was identified during initial screening, patients should receive firstline immunotherapy. It typically consists of corticosteroids, IVIG, or plasmapheresis. Half the patients will have symptom improvement within 4 weeks after tumor removal or first-line immunotherapy. Patients with poor improvement after first-line immunotherapy may benefit from second-line immunotherapy, which most commonly consists with rituximab and/or cyclophosphamide. About $80 \%$ may reach a mRS of 0-2 during the first 24 months with second-line immunotherapy. ${ }^{[2]}$

During the acute stage of the disease, many patients need to be hospitalized for at least 3 to 4 months and require extensive neurological monitoring. As for other autoimmune encephalitis disorders, common treatment protocols include 1 gm of IV methylprednisolone daily or $0.4 \mathrm{~g} / \mathrm{kg}$ of IVIg daily for 5 consecutive days. Plasma exchange given every other day for 10 to 14 days frequently proves beneficial for patients with severe disease or incomplete response to steroids and IVIg. Severely ill patients may require a combination of steroids, IVIg and plasma exchange therapy. Rituximab $375 \mathrm{mg} / \mathrm{m}^{2}$ IV weekly for 4 doses, with or without cyclophosphamide, should be considered where early trials of steroid, IVIg, and plasma exchange do not prove beneficial. Chronic maintenance therapy includes IVIg tapering schedule and/or $1 \mathrm{mg} / \mathrm{kg}$ per day of oral prednisone for 3 months followed by taper. In addition, azathioprine or mycophenolate mofetil is often added for maintenance therapy after completion of rituximab and/or cyclophosphamide therapy. Both azathioprine and mycophenolate mofetil can be used for chronic oral immunosuppressant therapy. Using slow conservative tapering permits the oral immunosuppressants to take effect before the steroids or IVIg are fully discontinued. Thus, initiation of a chronic oral immunosuppressant should overlap with the gradual taper of oral corticosteroids and/or IVIg infusions over a period of approximately 6 to 8 months, given that relapses frequently occur during rapid immunotherapy taper or discontinuation. ${ }^{[4,34]}$

Recovery from anti-NMDAR encephalitis occurs in the reverse order of symptom presentation as the immunological inhibition of NMDA receptors progressively decreases. Disease manifestations such as coma, autonomic instability, res- piratory depression and dyskinesia subside first. Patients may regain the capacity to follow simple commands and can have appropriate interactions before they recover verbal functions. Psychosis and agitation during this recovery period is not uncommon. Social behavior and executive functions are usually the last to improve. ${ }^{[23]}$

The risk of relapse is about $12 \%$, of which $33 \%$ may have multiple relapses. Patients without a tumor have a higher frequency of relapses than those with a tumor. Compared to the initial episode, most relapses are less severe, more frequently mono-symptomatic, and result in fewer admissions to the ICU. About 23\% may have relapses similar to the initial episode and a minority may have worse severity compared to the initial episode. The use of immunotherapy in the initial episode of encephalitis is associated with a lower frequency of relapses. Similarly, the use of second-line immunotherapy in patients without tumor has a relapse-decreasing effect. Introducing second-line immunotherapy during relapses also decreases the frequency of subsequent relapses. ${ }^{[2]}$

\section{Conclusion}

Our case highlights the fact that recognition of the clinical presentation of anti-NDMAR encephalitis requires understanding of the variability of prodromal symptoms, the evolution pattern of the disease, and the appropriate diagnostic studies available for its detection. Conscious use of simple diagnostic studies such as CSF analysis and EEG interpretation can clear a path for more specific serological tests, imaging studies, and empiric treatment. Generally speaking, the cost of diagnosis and management for patients with antiNMDAR encephalitis can be overwhelming. This presents a significant hurdle for diagnosis and a limiting factor in terms of financial load for both hospitals and patients. Appropriate use of current diagnostic studies can help tailor management and significantly decrease costs. Thus, judicious workup and management can help decrease unnecessary expenses, make an earlier diagnosis, and improve the quality of life of patients with anti-NMDAR encephalitis. This case serves to raise awareness of this serious medical condition, and to stress the point of how important is the early diagnosis of this disorder.

\section{Conflicts of InTEREST Disclosure}

The authors have declared no conflicts of interest.

\section{REFERENCES}

[1] Dalmau J, Gleichman AJ, Hughes EG, et al. Anti-NMDA-receptor encephalitis: Case series and analysis of the effects of antibodies. The Lancet Neurology. 2008; 7(12): 1091-8. https://doi.org/

Published by Sciedu Press
$10.1016 / \mathrm{S} 1474-4422(08) 70224-2$

[2] Titulaer MJ, McCracken L, Gabilondo I, et al. Treatment and prognostic factors for long-term outcome in patients with anti-NMDA receptor encephalitis: An observational cohort study. The Lancet 
Neurology. 2013; 12(2): 157-65. https ://doi.org/10.1016/S1 474-4422 (12)70310-1

[3] Wang W, Li J, Hu F, et al. Anti-NMDA receptor encephalitis: Clinical characteristics, predictors of outcome and the knowledge gap in southwest china. European Journal of Neurology. 2016; 23(3): 621-9. PMid:26563553 https : //doi.org/10.1111/ene.12911

[4] Rosenfeld MR, Dalmau J. Anti-NMDA-receptor encephalitis and other synaptic autoimmune disorders. Current treatment options in neurology. 2011; 13(3): 324-32. PMid:21298406 https ://doi.or $\mathrm{g} / 10.1007 / \mathrm{s} 11940-011-0116-\mathrm{y}$

[5] Thomas L, Mailles A, Desestret V, et al. Autoimmune N-methylD-aspartate receptor encephalitis is a differential diagnosis of infectious encephalitis. J Infect. 2014; 68(5): 419-25. PMid:24355654 https://doi.org/10.1016/j.jinf .2013.12.001

[6] Gable MS, Sheriff H, Dalmau J, et al. The frequency of autoimmune $\mathrm{N}$-methyl-D-aspartate receptor encephalitis surpasses that of individual viral etiologies in young individuals enrolled in the California encephalitis project. Clinical Infectious Diseases. 2012; 54(7): 899. PMid:22281844 https://doi.org/10.1093/cid/cir1038

[7] Schankin CJ, Kästele F, Gerdes LA, et al. New-onset headache in patients with autoimmune encephalitis is associated with anti-NMDAreceptor antibodies. Headache: The Journal of Head and Face Pain. 2016; 56(6): 995-1003. PMid:27241874 https://doi .org/10.1 $111 /$ head. 12853

[8] Suleiman J, Wright S, Gill D, et al. Autoantibodies to neuronal antigens in children with new-onset seizures classified according to the revised ILAE organization of seizures and epilepsies. Epilepsia. 2013; 54(12): 2091-100. PMid:24151870 https ://doi.org/10.1111/ epi. 12405

[9] Caplan J, Binius T, Lennon V, et al. Pseudopseudoseizures: Conditions That May Mimic Psychogenic Non-Epileptic Seizures. Psychosomatics. 2011; 52(6): 501-6. PMid:22054618 https : //doi .org/ $10.1016 / j \cdot$ psym. 2011.08 .004

[10] Sundararajan T, Tesar G, Jimenez X. Biomarkers in the diagnosis and study of psychogenic nonepileptic seizures: A systematic review. Seizure. 2016; 35(1): 11-22. PMid:26774202 https : //doi.org/10.1016/j.seizure.2015.12.011

[11] Gilmour G, Dix S, Fellini L, et al. NMDA receptors, cognition and schizophrenia - testing the validity of the NMDA receptor hypofunction hypothesis. Neuropharmacology. 2012; 62(3): 1401-12. PMid:21420987 https://doi.org/10.1016/j.neuropharm.2 011.03 .015

[12] Steiner J, Walter M, Glanz W, et al. Increased prevalence of diverse $\mathrm{N}$-methyl-D-aspartate glutamate receptor antibodies in patients with an initial diagnosis of schizophrenia: Specific relevance of IgG NR1a antibodies for distinction from N -methyl-D-aspartate glutamate receptor encephalitis. JAMA Psychiatry. 2013; 70(3): 271 PMid:23344076 https://doi.org/10.1001/2013.jamapsych iatry. 86

[13] Tsutsui K, Kanbayashi T, Tanaka K, et al. Anti-NMDA-receptor antibody detected in encephalitis, schizophrenia, and narcolepsy with psychotic features. BMC Psychiatry. 2012; 12: 37. PMid:22569157 https ://doi.org/10.1186/1471-244X-12-37

[14] Zandi MS, Irani SR, Lang B, et al. Disease-relevant autoantibodies in first episode schizophrenia. J Neurol. 2011; 258(4): 686-8. PMid:20972895 https : //doi.org/10.1007/s00415-010-578 8-9

[15] Prüss H, Finke C, Höltje M, et al. N-methyl-d-aspartate receptor antibodies in herpes simplex encephalitis. Ann Neurol. 2012; 72(6): 902911. PMid:23280840 https://doi.org/10.1002/ana. 23689

[16] Wei Y, Huang C, Liu C, et al. Peripheral neuropathy in limbic encephalitis with anti-glutamate receptor antibodies: Case report and systematic literature review. Brain and Behavior. 2017; 7(9): e00779. PMid:28948076 https : //doi.org/10.1002/brb3.779

[17] Malter MP, Elger CE, Surges R. Diagnostic value of CSF findings in antibody-associated limbic and anti-NMDAR-encephalitis. Seizure European Journal of Epilepsy. 2013; 22(2): 136-40. PMid:23318046 https://doi.org/10.1016/j.seizure.2012.12.013

[18] Wang R, Guan H, Ren H, et al. CSF findings in patients with anti-Nmethyl-d-aspartate receptor-encephalitis. Seizure. 2015; 29: 137-42. PMid:26076857 https://doi.org/10.1016/j.seizure. 2015 .04 .005

[19] Pillai SC, Hacohen Y, Tantsis E, et al. Infectious and autoantibodyassociated encephalitis: clinical features and long-term outcome. Pediatrics. 2015; 135(4): e974-84. PMid:25802349 https : //doi . org/10.1542/peds.2014-2702

[20] Oyanguren B, Sanchez V, Gonzalez FJ, et al. Limbic encephalitis: A clinical-radiological comparison between herpetic and autoimmune etiologies. European Journal of Neurology. 2013; 20(12): 1566-70. PMid:23941332 https ://doi.org/10.1111/ene.12249

[21] Baumgartner A, Rauer S, Mader I, et al. Cerebral FDG-PET and MRI findings in autoimmune limbic encephalitis: Correlation with autoantibody types. J Neurol. 2013; 260(11): 2744-53. PMid:23900756 https ://doi.org/10.1007/s00415-013-7048-2

[22] Heine J, Prüss H, Bartsch $T$, et al. Imaging of autoimmune encephalitis-Relevance for clinical practice and hippocampal function. Neuroscience. 2015; 309(11): 68-83. PMid:26012492 https : //doi.org/10.1016/j.neuroscience.2015.05.037

[23] Dalmau J, Lancaster E, Martinez-Hernandez E, et al. Clinical experience and laboratory investigations in patients with anti-NMDAR encephalitis. The Lancet Neurology. 2011; 10(1): 63-74. https : //doi.org/10.1016/S1474-4422(10)70253-2

[24] Veciana M, Becerra J, Fossas P, et al. EEG extreme delta brush: An ictal pattern in patients with anti-NMDA receptor encephalitis. Epilepsy \& Behavior. 2015; 49(1): 280-5. PMid:26071995 https://doi.org/10.1016/j.yebeh.2015.04.032

[25] Schmitt SE, Pargeon K, Frechette ES, et al. Extreme delta brush: A unique EEG pattern in adults with anti-NMDA receptor encephalitis. Neurology. 2012; 79(11): 1094-100. PMid:22933737 https ://doi.org/10.1212/WNL.0b013e3182698cd8

[26] Baysal-Kirac L, Tuzun E, Altindag E, et al. Are there any specific EEG findings in autoimmune epilepsies? Clinical EEG and Neuroscience. 2016; 47(3): 224-34. PMid:26240088 https : //doi .org/ $10.1177 / 1550059415595907$

[27] Foff EP, Taplinger D, Suski J, et al. EEG findings may serve as a potential biomarker for anti-NMDA receptor encephalitis. Clinical EEG and Neuroscience. 2017; 48(1): 48-53. PMid:27068513 https : //doi .org/10.1177/1550059416642660

[28] Baykan B, Tuncer OG, Vanli-Yavuz EN, et al. Delta Brush Pattern Is Not Unique to NMDAR Encephalitis: Evaluation of Two Independent Long-Term EEG Cohorts. Clinical EEG and Neuroscience. 2017: 155005941769316 PMid:29161898 https ://doi.org/10 $.1177 / 1550059417693168$

[29] Lancaster E, Leypoldt F, Titulaer MJ, et al. Immunoglobulin G antibodies to the N-Methyl-D-aspartate receptor are distinct from immunoglobulin A and immunoglobulin M responses. Ann Neurol. 2015; 77(1): 183. PMid:25048078 https://doi.org/10.1002/ ana. 24233

[30] Hammer C, Stepniak B, Schneider A, et al. Neuropsychiatric disease relevance of circulating anti-NMDA receptor autoantibodies depends on blood-brain-barrier integrity. Molecular Psychiatry. 2013; 19(1): 1143-9. PMid:23999527 
[31] Dahm L, Ott C, Steiner J, et al. Seroprevalence of autoantibodies against brain antigens in health and disease. Ann Neurol. 2014; 76(1): 82-94. PMid:24853231 https://doi .org/10.1002/ana. 24189

[32] Gresa-Arribas N, Titulaer MJ, Torrents A, et al. Diagnosis and significance of antibody titers in anti-NMDA receptor encephalitis, a retrospective study. Lancet Neurology. 2014; 13(2): 167-77. https ://doi.org/10.1016/S1474-4422(13)70282-5
[33] Titulaer MJ, Soffietti R, Dalmau J, et al. Screening for tumors in paraneoplastic syndromes: Report of an EFNS task force. European Journal of Neurology. 2011; 18(1): e3. PMid:20880069 https://doi.org/10.1111/j.1468-1331.2010.03220.x

[34] McKeon A. Paraneoplastic and Other Autoimmune Disorders of the Central Nervous System. The Neurohospitalist. 2013; 3(2): 53-64. PMid:23983888 https://doi.org/10.1177/19418744124533 39 